Pub. Mat, UAB

Vol. 29 No 1 Abril 1985

\title{
A THEOREM ON SCHAUDER DECOMPOSITIONS IN BANACH SPACES
}

Migue1 A. Ariño

Abstract. In this paper we prove that in a Banach space all Schauder decompositions are shrinking iff all schauder decompositions are boundediy complete.

\section{Definitions and preliminary results}

A sequence $\left(x_{n}\right)_{n=1}^{\infty}$ in a Banach space $x$ is called a Schauder basis if for every $x \in X$ there exists a unique sequence $\left(\alpha_{n}\right)_{n=1}^{\infty}$ in $R$ such that $x=\sum_{n=1}^{\infty} a_{n} x_{n}$, and this series converges with respect the norm of $x$. A sequence $\left(y_{n}\right)_{n=1}^{\infty}$ is called a basic sequence if it is a basis of his closed linear span.

A Schauder decomposition of $x$ is a sequence $\left(x_{i}\right)_{i=1}^{\infty}$ of closed subspaces of $x$ such that for every $x$ in $x$ there exists a unique sequence $\left(x_{i}\right)_{i=1}^{\infty}$ with $x_{i} \in x_{i}$ for all $i$ and $x=\sum_{i=1}^{\infty} x_{i}$. Every Schauder decomposition of $x$ is related with a sequence of continuous frojections $\mathrm{P}_{\mathrm{n}}: \mathrm{X} \longrightarrow \mathrm{X}$ defined by

$$
P_{n}(x)=P_{n}\left(\sum_{i=1}^{\infty} x_{i}\right)=\sum_{i=1}^{n} x_{i}
$$

In all this paper, the linear span of an element $x \in \mathrm{x}$ is denoted by $\{x\}$ and the closed linear span of the subspaces $\left(x_{i}\right)_{i=n}^{m}$ $(1 \leq n<m \leq \infty)$ is denoted by $\left[x_{i}\right]_{i=r}^{m}$.

The following theorem characterizes the Schauder decompositions and it can be found in [5]. 
1. Theorem: Let $X$ be a Eanach space and $\left(x_{n}\right)_{n=1}^{\infty}$ a sequence of closed subspaces of $x$. The following are equivalent:

i) $\left(X_{n}\right)_{n=1}$ is a Schauder decomposition of $x$.

ii) There exists a sequence $\left(P_{n}\right)_{n=1}^{\infty}$ of continuous projections $P_{n}$ : $x \rightarrow\left[x_{i}\right]_{i=1}^{n}$ such that $\quad P_{n} P_{m}=P_{m i n}(m, n)$. and $\lim _{n \rightarrow \infty} P_{n}(x)=x$ for every $x$ in $x$.

iii) There exists a sequence $\left(\mathrm{P}_{n}\right)_{n=1}^{\infty}$ of continuous projections $\mathrm{P}_{n}$ : $x \longrightarrow\left[x_{i}\right]_{i=1}^{n} \quad$ such that $P_{n} P_{m}=P_{\min (m, n)}$ and $\left(P_{n}\right)_{n=1}^{\infty}$ is uniformily bounded.

To sup $\left\|P_{n}\right\|$ is called norm of the decomposition.

A Schauder decomposition $\left(x_{n}\right)_{n=1}^{\infty}$ in a Banach space $x$ is called boundedly complete if for every sequence $\left(x_{n}\right)_{n=1}^{\infty}$ with $x_{n} \in x_{n}$ such that $\sup _{n}\left\|\sum_{i=1}^{n} x_{i}\right\|<\infty$, the sequence $\left(\sum_{i=1}^{n} x_{i}\right)_{n=1}^{\infty}$ converges towards an element $x$ in $x$. And it is called shrinking if for every $x^{*} \in X, \lim _{n \rightarrow \infty} \| x_{n}^{*}=0$, where

$$
\left\|x^{*}\right\|_{n}=\sup \left\{\left|x^{*}(x)\right| \text { with } x \in\left[x_{i}\right]_{i=n+I}^{\infty} \text { and }\|x\| \leq 1\right\} \text {. }
$$

Boundedly complete and shrinking basis and basic sequences are defined in a similar way.

Singer (cf. [6]) has proved that in a Banach space all basic sequences are boundedly complete if and only if all basic sequences are shrinking. Afterwards Zippin (cf. [7] and [3]) proved a similar theorem for Schauder basis of $x$. Our purpose in this paper is to prove that in a Banach space all Schauder decompositions are boundedly complete iff all Schauder decompositions are shrinking. 
If $x$ is a locally bounded F-space, then there exists $p(0<p<1)$ such that the topology of $x$ is originated by a p-norm. In this case $x$ is called p-Banach space (cf. [1] and [4]). Let $X$ be a p-Banach space such that $x$ separates points of $x$ and let $J: X \longrightarrow x^{* *}$ be the canonical imbedding of $x$ into its bidual. We define in $x$ the norm H. $\|^{* *}$ :

$$
\|x\|^{* *}=\|\mathrm{J}(\mathrm{x})\| \quad \text { if } \quad x \in X \text {. }
$$

The Mackey topology of $x$ is criginated by this norm (cf. [2]) and it is called the Mackey norm of $x$. The Mackey completion of $X$ is denoted by $\overline{J(x)}$.

All the above definitions for Banach spaces can be extended to p-Banach spaces.

\section{Shrinking and boundedly complete Schauder decomposition.}

2. Lemma. Let $\left(x_{n}\right)_{n=1}^{\infty}$ be a Schauder decomposition of a Banach space $x$ and let $\left(P_{n}\right)_{n=1}^{\infty}$ be its sequence of projections. We suppose that each $X_{n}$ admits a topological decomposition $X_{n}=Y_{n} \oplus Z_{n}$. The following are equivalent:

i) $\left(Y_{1}, Z_{1}, \ldots, Y_{n}, Z_{n}, \ldots\right)$ is a schauder decomposition of $X$.

ii) If $A_{n}$ is the continuous projection from $X_{n}$ into $Y_{n}$, then $\sup _{n}\left\|A_{n}\right\|<\infty$.

Proof: $i \Rightarrow$ ii. If $\left(Q_{n}\right)_{n=1}^{\infty}$ is the sequence of projections of $\left(Y_{1}, Z_{1}, \ldots\right.$ $\left.\ldots, Y_{n}, Z_{n}, \ldots\right)$, as $A_{n}=Q_{2 n-1 \mid x_{n}}$, the statement ii is proved. 
ii $\Rightarrow$ i. If $\sup _{n}\left\|A_{n}\right\|<\infty$, we define

$$
\begin{aligned}
& Q_{2 n}=P_{n} \\
& Q_{2 n+1}=P_{n}+A_{n}\left(P_{n}-P_{n-1}\right) \quad n>1 \\
& Q_{1}=A_{1} P_{1}
\end{aligned}
$$

and thus $\left(Q_{n}\right)_{n=1}^{\infty}$ is a uniformly bounded sequence of projections which defines the decomposition $\left(Y_{n}, Z_{n}\right)_{n=1}^{\infty}$ because of theorem 1 .

/

Remark that if any of the grevious subspaces is 0 , it must be taken away in the decomposition.

3. Corollary. Let $\left(x_{n}\right)_{n=1}^{\infty}$ be a Schauder decomposition of a Banach space $X$ and $\left(x_{n}\right)_{n=1}^{\infty}$ a normalized sequence in $X$ with $x_{n} \in X_{n}$. For every $n$ there exists an hyperplane $w_{n}$ of $x_{n}$ such that $\left(\left[x_{1}\right], w_{1}, \ldots,\left[x_{n}\right], w_{n}\right.$, ...) is a schauder decomposition of $x$.

Proof: As $\left\|x_{n}\right\|=1$, we can define $A_{n}(x)=u_{n}^{*}(x) x_{n}$, where $u_{n}^{*} \in x_{n}^{*}$ and $u_{n}^{*}\left(x_{n}\right)=\left\|u_{n}^{*}\right\|=1$.

4. Lemrna. Let $x$ be a Banach space and a Schauder decomposition of the form $\left(\left[y_{1}\right], w_{1}, \ldots,\left[y_{n}\right], w_{n}, \ldots\right)$ where $\left(y_{n}\right)_{n=1}^{\infty}$ satisfies $\inf _{n}\left\|y_{n}\right\|=C>0$ and $\sup _{n}\left\|\sum_{i=1}^{n} y_{i}\right\|=M<\infty$. We define the sequence $\left(v_{n}\right)_{n=1}^{\infty}$ by $v_{n}=\sum_{i=1}^{n} y_{i}$. Then $\left(\left[v_{1}\right], w_{1}, \ldots,\left[v_{n}\right], w_{n}, \ldots\right)$ is a Schauder decomposition of $X$.

Proof: Let $\left(P_{n}\right)_{n=1}^{\infty}$ be the sequence of projections of $\left(x_{n}\right)_{n=1}^{\infty}$ and let $K$ be its norm. Each $P_{2 n-1}-P_{2 n-2}$ (the projection over $\left[y_{n}\right]$ ) is originated by a $y_{n}^{*} \in x^{*}$ according to 


$$
\left(P_{2 n-1}-P_{2 n-2}\right)(x)=y_{n}^{*}(x) y_{n} \text { if } \quad x \in X .
$$

and thus

i) $y_{n}^{*}\left(y_{m}\right)=\delta_{n, m}$

ii) $\left\|y_{n}^{*}\right\|\left\|y_{n}\right\| \leq 2 K$ for every $t$, and

iiii) $\left.y_{n}^{*}\right|_{m}=0$ for every $n$ and $m$.

As $\inf _{n}\left\|y_{n}\right\|=C>0$, from ii) we obtain that $\sup _{n}\left\|y_{n}^{*}\right\| \leq 2 K / C$. Let $\left(v_{n}^{*}\right)_{n=1}^{\infty}$ be defined be $v_{n}^{*}=y_{n}^{*}-y_{n+1}^{*}$. It is easy to check that $v_{n}^{*}\left(v_{m}\right)=\delta_{n, m}$.

We define the sequence of projections by

$$
\begin{aligned}
& A_{2 n}(x)=\sum_{k=1}^{n}\left(P_{2 k}-P_{2 k-1}\right)(x)+\sum_{k=1}^{n} v_{k}^{*}(x) v_{k} \\
& A_{2 n+1}(x)=A_{2 n}(x)+v_{n+1}^{*}(x) v_{n+1} .
\end{aligned}
$$

Because of the theorem 1 we only need to prove that $\left(A_{n}\right)_{n=1}^{\infty}$ is uniformly bounded, and, because of the last considerations, it shall be proved if we prove that $\sup _{n}\left\|A_{2 n}\right\|<\infty$ :

$$
\begin{aligned}
&\left\|A_{2 n}(x)\right\|=\left\|P_{2 n}(x)-\sum_{k=1}^{n} y_{k}^{*}(x) y_{k}+\sum_{k=1}^{n}\left(y_{k}^{*}(x)-y_{k+1}^{*}(x)\right)\left(\sum_{i=1}^{k} y_{i}\right)\right\| \leq \\
& \leq x\|x\|+\left\|-\sum_{k=1}^{n} y_{k}^{*}(x) y_{k}+\sum_{k=1}^{n}\left(y_{k}^{*}(x)-y_{n+1}^{*}(x)\right) y_{k}\right\| \leq \\
& \leq k\|x\|+\left\|y_{n+1}^{*}\right\|\|x\|\left\|\sum_{k=1}^{n} y_{k}\right\| \leq\left(k+\frac{2 k}{C} M\right)\|x\| .
\end{aligned}
$$


5. Lemma . Let $x$ be a Banach space and $\left(\left[y_{1}\right], w_{1}, \ldots,\left[y_{n}\right], w_{n}, \ldots\right)$ a Schauder decomposition of $x$, where $\left(y_{n}\right)^{\infty}$ satisfies $\sup _{n}\left\|y_{n}\right\|=M<\infty$. we define the sequence $\left(v_{n}\right)_{n=1}^{\infty}$ by $v_{1}=y_{1}$ and $v_{n}=y_{n}-y_{n-1}$. Then the following are equivalent:

i) $\left(\left[v_{1}\right], w_{1}, \ldots,\left[v_{n}\right], w_{n}, \ldots\right)$ is a Schauder decomposition of $x$.

ii) There exists $x^{*} \in x^{*}$ such that

a) $x^{*}\left(y_{n}\right)=1$ for every $n$

b) $\left.x^{*}\right|_{m}=0$ for every $m$

Proof: If, for every $n$, there is a continuous projection from $x$ into $\left[v_{n}\right]$ parallel to the other subspaces, the existence of $x^{*} \in X^{*}$ satisfying a) and b) is necessary. We suppose that there exists a such $x^{*}$. We define $\left(y_{n}^{*}\right)_{n=1}^{\infty}$ as in the preceding lemma, and if we consider the sequence

$$
v_{1}^{*}=x^{*} \text { and } v_{n}^{*}=x^{*}-\sum_{k=1}^{n-1} y_{k}^{*}
$$

the orthogonal relations $v_{n}^{*}\left(v_{m}\right)=\delta_{n, m}$, hold.

Let $\left(A_{n}\right)_{n=1}^{\infty}$ be a sequence of projections as in the preceding lemma. We must prove that $\sup _{n}\left\|A_{n}\right\|<\infty$. For every $m,\left.x^{*}\right|_{w_{m}}=0$, and hence $x^{*}(x)=\sum_{n=1}^{\infty} y_{n}^{*}(x)$ for every $x$ in $x$ and so $\left(v_{n}^{*}\right)_{n=1}^{\infty}$ converges weakly to 0 and $\sup _{n}\left\|\sum_{k=1}^{n} y_{k}\right\|=M_{1}<\infty . \quad$ 1so $\sup _{n}\left\|v_{n}\right\| \leq 2 M$, again we must only prove that $\sup _{n}\left\|A_{2 n}\right\|<\infty$ :

we have

$\sum_{k=1}^{n} v_{k}^{*}(x) v_{k}=x^{*}(x)+\sum_{k=2}^{n} x^{*}(x)\left(y_{k}-y_{k-1}\right)-\sum_{k=2}^{n}\left[\left(\sum_{i=1}^{k-1} y_{i}^{*}\right)(x)\right]\left(y_{k}-y_{k-1}\right)=$ 


$$
\begin{aligned}
& -x^{*}(x) y_{1}+x^{*}(x)\left(y_{n}^{*}-y_{1}\right)-\sum_{k=1}^{n-1} y_{k}^{*}(x) y_{n}+\sum_{k=1}^{n-1} y_{k}^{*}(x) y_{k}= \\
& =\sum_{k=1}^{n} y_{k}^{*}(x) y_{k}+x^{*}(x) y_{n}-\sum_{k=1}^{n} y_{k}^{*}(x) y_{n}
\end{aligned}
$$

and thus

$$
A_{2 n}(x)=P_{2 n}(x)+x^{*}(x) y_{n}-\sum_{k=1}^{n} y_{k}^{*}(x) y_{n}
$$

And finally:

$$
\left\|A_{2 n}(x)\right\| \leq\left\|P_{2 n}\right\|\|x\|+\|x *\|\|x\|\left\|y_{n}\right\|+\left\|\sum_{k=1}^{n} y_{k}^{*}\right\|\|x\|\left\|y_{n}\right\|
$$

and

$$
\left\|A_{2 n}\right\| \leq K+M\left\|x^{*}\right\|+M_{1} M
$$

Now we can prove the main result:

6. Theorem. Let $\times$ be a Banach space. The following statements are equivalent:

i) All Schauder decompositions of $x$ are shrinking

ii) All Schauder decorapositions of $X$ are boundediy complete

Proof. $i \Rightarrow i j$. Let $\left(x_{n}\right)_{n=1}^{\infty}$ be a non boundedly complete Schauder decomposition of $x$. There exists then a sequence $\left(x_{i}\right)_{i=1}^{\infty}$ with $x_{i} \in x_{i}$ such that $\sup _{n} \| \sum_{i=1}^{n} x_{i}=1$ and $\left(\sum_{i=1}^{n} x_{i}\right)_{n=1}^{\infty}$ is not a Cauchy sequen$c e$, and thus, there exist $E$ and a strictly increasing sequence $\left(m_{k}\right)_{k=1}^{\infty}$ such that $\varepsilon<\left\|\sum_{i=m_{k-1}+1}^{m_{k}} x_{i}\right\| \leq 2$ for every $k$. We desine $Y_{k}=\left[x_{i}\right]_{i=m_{k-1} m_{k}+1}^{n}$ and $y_{k}=\sum_{i=m_{k-1}+1}^{m_{k}} x_{i}$ if $k \geq 1$. $\left(Y_{k}\right)_{k=1}^{\infty}$ is a Schauder decomposition of $x$ with $y_{k} \in Y_{k}$. Because of the corollary 3 , for each $k$ there exists a hyperplane $w_{k}$ of $y_{k}$ such that $\left(\left[y_{1}\right], w_{1}, \ldots\right.$ 
$\left.\cdots,\left[y_{n}\right], w_{n}, \ldots\right)$ is a schauder decomposition. Because of the lemma 4 , the sequence $\left(v_{k}\right)_{k=1}^{\infty}$ defined by $v_{n}=\sum_{i=1}^{n} y_{i}$ originates the schauder decomposition $\left(\left[v_{1}\right], w_{1}, \ldots,\left[v_{n}\right], w_{n}, \ldots\right)$ which is not shrinking because of $y_{1}^{*}\left(v_{k}\right)=1$ for every $k \geq 1$.

ii $\Rightarrow i$. Let $\left(x_{n}\right)_{n=1}^{\infty}$ be a non shrinking schauder decomposition of $x$. There exist then $x^{*} \in x$ with $\left\|x^{*}\right\|=1, \quad \varepsilon>0$, a strictly increasing sequence of index $\left(m_{k}\right)_{k=1}^{m}$ and a sequence $\left(y_{k}\right)_{k=1}^{m}$ with $y_{k} \in Y_{k}=\left[x_{i}\right]_{i=m_{k-1}}^{m_{k}}$ such that:
a) $1 \leq\left\|y_{n}\right\| \leq 1 / \varepsilon$
b) $x^{*}\left(y_{n}\right)=1$.

We can choose the hyperplane $W_{k}=Y_{k} \cap K e r x^{*}$ and using the lemma 5, if $v_{1}=y_{1}$ and $v_{n}=y_{n}-y_{n-1}$, then $\left(\left[v_{1}\right], w_{1}, \ldots,\left[v_{n}\right], w_{n}, \ldots\right)$ is a Schauder decomposition of $x$ which is not boundedly complete because of

$$
\left\|\sum_{k=2}^{n} v_{k}\right\|=\left\|\sum_{k=2}^{n}\left(y_{k}-y_{k-1}\right)\right\|=\left\|y_{n}-y_{1}\right\| \leq 2 / \varepsilon
$$

while

$$
\left\|v_{k}\right\|=\left\|y_{k}-y_{k-1}\right\| \geq \frac{1}{k}\left\|y_{k-1}\right\|
$$

where $x$ is the norm of $\left(x_{n}\right)_{n=1}^{\infty}$.

With certain modifications, this theorem has an extension to p-Banach spaces (if its dual separates points). The Mackey topology of this spaces plays an important role in this extension. We need before a definition: we shall say that a Schauder decomposition $\left(x_{n}\right)_{n=1}^{\infty}$ in a p-Banach space is an almost boundedly complete decomposition if for every sequence $\left(x_{n}\right)_{n=1}^{\infty}$ with $x_{n} \in x_{n}$ such that $\sup _{n}\left\|\sum_{k=1}^{n} x_{k}\right\|<\infty$, the sequence $\left(\sum_{k=1}^{n} x_{k}\right)_{n=1}^{\infty}$ converges in $\left(\overline{J(x)},\|.\|^{* *}\right)$. 
We must point out that if $\left(x_{n}\right)_{n=1}^{\infty}$ is boundedly complete then it is also almost boundedly complete. Almost boundedly complete bas is of $X$ are defined in a similar way.

7. Theorem. Let $X$ be a p-Banach space. The following are equivalent:

i) All Schauder decompositions of $X$ are shrinking.

ii) All Schauder decompositions of $X$ are almost boundedly complete.

Proof: Similar to the proof of Theorem 6, and it can be found in [2]

I would like to express my acknowledgment to my research supervisor Profossor M. A. Canela for his help and encouragement ciuring my work and for the diffusion given to this paper in his lectures.

\section{References}

(1) T. Aoki. Locally bounded linear topological spaces, Proc. Imperial Acad. (Tokyo) 18, (1942), pp. 588-594.

[2] M.A. Ariño. Bases y descomposiciones de Schauder en espacios p-Banach, Thesis, Barcelona Univ. Barcelona (1984).

(3) D.V. Dulst, Reflexive and super-reflexive Banach spaces, Mathematical centre, Amsterdam (1978).

[4] S. Rolewicz, on a certain class of linear metric spaces, Bull. Acad. Polon. Sci. 5(1957), pp. 471-473.

(5] I. Singer, Bases in Banach spaces II. Springer Verlag, Berlin (1970).

(6) I. Singer, Basic sequences and reflexivity of Banach spaces. Studia Math. 21, (1962), pp. 359-369.

[7] M. Zippin, A remark on bases and reflexivity in Banach spaces. Israel J. Math. 6, (1968), pp. 74-79.

Rebut el 19 de novembre del 1984

Facultad de Matemáticas

Dpto. de Teoria de Funciones

Universidad de Barcelona

Gran Via, 585

Barcelona

SPAIN 